\title{
Influence of Microscopic Effects on the Static Tensile Strength of Gray Cast Iron HT200 Specimens
}

\author{
L. Fan, ${ }^{1}$ H. B. Hu, ${ }^{1}$ X. S. Tang, ${ }^{2}$ J. J. He, ${ }^{1}$ and W. Chen ${ }^{3}{ }^{3}$ \\ ${ }^{1}$ Changsha Road and Bridge Construction Co.,Ltd, Changsha, Hunan 410114, China \\ ${ }^{2}$ School of Civil Engineering and Architecture, Changsha University of Science and Technology, Changsha, Hunan 410114, China \\ ${ }^{3}$ Key Laboratory of Disaster Prevention and Structural Safety of Ministry of Education, Guangxi University, \\ Nanning 530004, China \\ Correspondence should be addressed to W. Chen; w.chen@st.gxu.edu.cn
}

Received 16 October 2020; Revised 26 December 2020; Accepted 18 January 2021; Published 5 February 2021

Academic Editor: Massimiliano Barletta

Copyright (c) 2021 L. Fan et al. This is an open access article distributed under the Creative Commons Attribution License, which permits unrestricted use, distribution, and reproduction in any medium, provided the original work is properly cited.

Gray cast iron HT200 material is a kind of pearlitic gray cast iron. Flake graphite in the gray cast iron greatly destroys the integrity of the matrix and affect the static strength. The influence of microscopic effects on the tensile static strength of gray cast iron HT200 specimens is investigated. The microstructures are observed by the scanning electron microscope. The failure tests are done under the static loads for the cylinder specimens of gray cast iron HT200. Then, an energy density zone (EDZ) model is applied to the simulation of the fracture process of the specimens. The energy density zone model is a macro/micro-trans-scale crack growth model that can depict a fracture process from an initial microdefect at the microscale to the final break at the macroscale. Three scale transitional functions as well as the size of the initial microdefect in the model represent the microscopic effects in a fracture process. Three scale transitional functions are speculated in view of the physical failure mechanisms. Two other material parameters in the model are determined from the test data. Thereby, the fracture process of gray cast iron specimens is numerically simulated, and the static strength values are calculated. The calculated values of static strength of gray cast iron specimens are identical to the test values. It is seen that the energy density zone model can accurately describe a fracture process of brittle materials like gray cast iron. In addition, the calculated results show that the microscopic effects did affect the static strength of gray cast material.

\section{Introduction}

Gray cast iron HT200 is a kind of pearlitic gray cast iron with high strength and hardness. The microstructures mainly consist of a pearlitic matrix and Flake graphite. It is widely used for important castings in machines such as cylinder stator, cylinder block, piston, wheel gear, engine base, and pressure valve body. The mechanical property of gray cast iron materials depends on the matrix structures and the plumbaginous morphologies. Flake graphite in the matrix cuts the matrix, induces the stress concentration at the graphite sharp corners, and further affects the static strength as well as the fatigue behavior.

So, it is significant to understand the influence of microscopic effects on the mechanical property of gray cast iron or other materials, for example, superalloy [1-3]. Many investigations on gray cast iron of microscopic influences have been done in the past such as in [4-12]. For instance, the fracture of a compressor rotor manufactured from gray cast iron was investigated [4]. In order to study the causes of the fracture, specimens prepared from the damaged rotor were subjected to experiments such as hardness and Charpy impact tests. The effect of microstructure on the fracture was also considered. Geometrical effects on fracture toughness of lamellar gray cast iron were addressed [5]. The graphite lamellae act as stress concentrators that produce microcracking of the lamellae or interface debonding at a very low stress level. Geometrical effects on different fracture toughness parameters were analyzed. The tested material was a lamellar gray cast iron with A-type graphite, size 4 , and 
ferritic-pearlitic matrix. High-temperature low cycle fatigue behavior of a gray cast iron or engine cylinder blocks was studied [6]. Transmission electron microscopy analysis suggested that cyclic softening was caused by the interactions of dislocations at $423 \mathrm{~K}$, such as cell structure in ferrite, whereas cyclic softening was related to subgrain boundaries and dislocation climbing at $523 \mathrm{~K}$. The initial multiaxial yield behavior of three different gray cast iron materials with lamellar shaped graphite inclusions was numerically simulated by means of the finite element method [7]. Volume elements including the real microstructure of the materials were employed in the simulations. The microstructure, phase composition, and microhardness of rapidly solidified gray cast iron BS1452 Grade 250 were compared against the conventionally solidified alloy [8]. Microstructure evaluations were made by optical and scanning electron microscopy, while XRD was used for the identification and analysis of evolved phases. Fracture behavior under torsion of notched round bars made of gray cast iron was analyzed [9]. Experimental results of 25 gray cast iron V-notched specimens subjected to torsion were presented. The SED (Strain Energy Density) criterion is used for the analysis of these elastic-plastic notched specimens. A notable work was done to correlate microstructure and mechanical properties of pearlitic gray cast iron [10]. The material under study was extracted from identical sand-cast parts produced by three different foundries. Laboratory tests show that mechanical properties, as tensile and fatigue strength, of gray cast iron of the same grade vary from foundry to foundry. A noticeable statistical data scatter is found also, depending on the location of the castings from which the specimens are machined. The cause of this behavior is ascribed to the inhomogeneity that characterizes the gray cast iron microstructure. A methodology for the fatigue design of notched castings in gray cast iron was developed [11]. The work consisted of an experimental procedure and a numerical design activity. The implication was that cracks and localized damage begin to appear in the microstructure of gray cast iron at sharp notches from the first cycles of loading. Casting experiments were carried out to produce gray cast irons with different compositions [12]. Their influence on the development of microstructure and mechanical properties was assessed. It was found that microstructures ranging from fully pearlitic to an intimate mixture of retained austenite and bainitic ferrite, termed ausferrite, were produced and a reasonable linear correlation was observed between ausferrite volume fraction and strength.

After a short reference review, it is clear that the mechanical property of gray cast iron materials indeed depends on their microstructures. Definitely, there is a correlation between the scatter of macroscopic mechanical parameters and the microscopic effects. The key point of the problem is the link between two different scales from microscale to macroscale. The energy density theory provides a powerful analytical tool for multiscale problems. Material failure is a typical multiscale problem $[13,14]$ because the failure first triggers from an initial microdefect zone and finally comes to the final break that passes two different scales from microscale to macroscale. In recent years, an energy density zone model has been developed to depict the scale transitional characteristics in a failure process [15-24] and has been successfully applied to the simulation of fatigue failure problems [25-28]. Initially, the model is called the restraining stress zone (RSZ) model. Sih suggested that the model is better called the energy density zone (EDZ) model [26]. Here, the key idea is that the nonequilibrium and nonhomogeneity (NENH) process of material failures can be divided into many small time steps. In each small time step, the material can be thought of as equilibrium and homogeneity such that the model can be solved by the classical continuum mechanics with the omission of NENH effects in this small time step. In the next step, the material property changes with the shift of the analytical scale. In this way, the NENH effects can be taken into account in the EDZ model.

The previous work is for the fatigue failure problems [25-28]. This work focuses on the fracture problem of gray cast iron materials under static loads. The aim is to show how the microscopic effects are depicted in the formulation and how the microstructures affect the material static strength.

\section{Loading Test and Static Strength}

The tensile test equipment is shown in Figure 1. CTM8000 series tensile testing machine fully considers the relevant principles of modern industrial design and ergonomics. The shell adopts an alloy frame structure to enhance the stiffness coefficient and double-layer metal baking paint on the surface, so as to prolong the service life of the host and beautify the appearance. The servo motor drive system is used to drive the beam to move up and down to realize the test loading process. It is mainly suitable for testing and analyzing the mechanical properties of various metal, nonmetallic, and composite materials with a test load of less than $50 \mathrm{kN}$. It has accurate loading speed and force range and has higher precision and sensitivity for the measurement and control of the load, deformation, and displacement and can also carry out constant speed. It has the function of low cycle load cycle, deformation cycle, and displacement cycle. According to GB, ISO, JIS, ASTM, DIN, and other international standards, the maximum force, tensile strength, bending strength, compressive strength, modulus of elasticity, elongation at break, yield strength, and other parameters can be obtained, and the test data curve can be superimposed, analyzed, stored, printed, and plotted, and a complete report sheet can be printed for process adjustment and production control.

The SEM equipment is shown in Figure 2. SS-150 series desktop scanning electron microscope has the advantages of small size and small space occupation. It can clearly observe the microstructure of cast iron samples by using a double detector of secondary electron and backscattered electron, magnification of 30 150000 X, and resolution of $5 \mathrm{~nm}$. The EDS can be used to analyze the element composition. It has powerful software function, simple operation, and easy maintenance.

Six tensile specimens of gray cast iron HT200 are as shown in Figure 3. The uniaxial tensile tests were carried out in the laboratory under static loading. The results are listed in Table 1. 


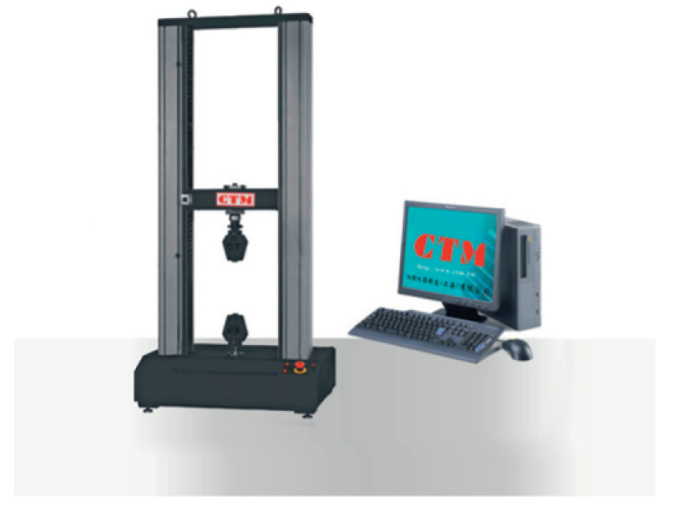

Figure 1: Tensile test device (CTM8000).

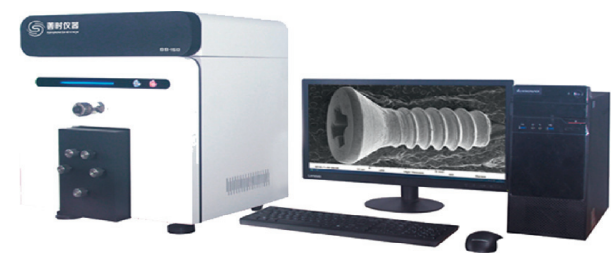

Figure 2: Scanning electron microscope device (SS-150).

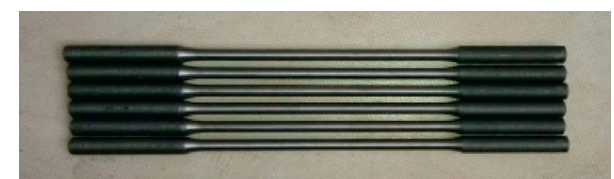

Figure 3: Photo of uniaxial tensile specimens of gray cast iron HT200.

The values of static strength are also illustrated in Figure 4. It is seen that the value of extreme strength for six specimens is different from specimen to specimen even if the same size and shape specimens are made from the same material under the same test condition. The scatter of test data cannot be completely attributed to test machine errors because the test system would not cause such a large dispersion. It can be speculated that this dispersion of test data is mostly produced by the material microscopic effects because the microstructure of specimens is different from one to another.

A microscopic observation was done for the gray cast iron HT200 material. A photo of the fracture section of the specimen is illustrated in Figure 5. A SEM photo with the amplification of 1000 times to show the microstructures is shown in Figure 4. Note that there is a microscopic defect with the length of the dozens of microns in the central area of the photo as shown in Figure 6.

\section{Energy Density Zone Model and Formulation}

Gray cast iron is a kind of brittle material and its fracture process is plotted in Figure 7. Under the uniaxial tension, the fracture process makes onsets from a microdefect as shown in Figure 7(a). Then, the microcrack forms and grows referring to Figure $7(\mathrm{~b})$ when the loading begins. With the further increment of static loads, the macrocrack forms and grows to the final fracture as indicated in Figure 7 (c).

This fracture process in the case of static loading can be simulated by the energy density zone (EDZ) model as plotted in Figure 8. For more information on the EDZ model, please refer to the past work [11, 22-25]. Here, a short summary is necessary.

Time $t$ should be understood as the generalized time, such as the number of load cycles for fatigue failure problems or the loading steps for static failure problems. Material damage can be represented by an energy density zone where the distributed restraining stresses prevail. The strain energy density factor dominates the crack propagation from microscale to macroscale. This is the reason that the material damage zone with the length $a$ is called the energy density zone. The case of $\sigma^{*}=\sigma_{0} /$ $\sigma=0$ denotes that the material is completely destroyed and a macrocrack forms. $\sigma_{0}$ is the mean value of restraining stresses distributed in EDZ. Apparently, the restraining stress need not be uniformly distributed in EDZ that depends on the material damage condition. $\sigma$ is the applied stress. On the other side, the case of $\sigma^{*}=\sigma_{0} / \sigma=1$ corresponds to the fact that the material has no damage. Therefore, the restraining stress ratio $\sigma^{*}=\sigma_{0} / \sigma$ can serve as a damage variable. It should be emphasized that all quantities shown in Figure 6 vary with time $t$. This allows the EDZ model to depict the scale shift from microscale to macroscale and capture NENH effects in a fracture process. With the increment of loading steps, the length $a$ of EDZ gradually increases. Meanwhile, the ratio $\sigma^{*}=\sigma_{0} / \sigma$ decreases representing the increase of material damage degree in EDZ. When the size $a$ comes to a macroscopic scale (i.e., the millimeter level) and the restraining stresses in EDZ vanish, a macrocrack forms that can be seen by naked eyes. The further growth of macrocrack will lead to the final fracture of specimens. In such a way, the EDZ model as illustrated in Figure 6 can simulate the whole failure process of specimens from the initial microdefect to the final break. Note that a microscopic crack tip damage zone is attached to the front of EDZ as shown in Figure 8. This treatment can allow the microscopic geometric parameters to enter the formulation.

In the case of uniform distribution of restraining stresses in the energy density zone (i.e., the material damage zone), the problem represented by Figure 8 has been analytically solved by the complex function approach [19]. In the case of the linear distribution of restraining stresses in EDZ, the analytical solution has also been obtained [21]. It can be concluded that for the general cases of nonuniform distribution of restraining stresses, the analytical expression of transscale strain energy density factor $S_{\text {micro }}^{\text {macro from microscale }}$ to macroscale can be written in the form of [11]

$$
\begin{aligned}
\mu^{*} & =\frac{\mu_{\text {micro }}}{\mu_{\text {macro }}}, \\
\sigma^{*} & =\frac{\sigma_{0}}{\sigma}, \\
d^{*} & =\frac{d}{d_{0}},
\end{aligned}
$$$$
S_{\text {micro }}^{\text {macro }}=B a \sigma^{2} \mu^{*}\left(1-\sigma^{*}\right)^{2} \sqrt{d^{*}},
$$ 
TABLE 1: Test values of extreme strength of gray cast iron HT200 specimens.

\begin{tabular}{lcccc}
\hline Specimen number & Diameter $\Phi(\mathrm{mm})$ & Section area $A(\mathrm{~mm})^{2}$ & Extreme force $F_{b}(\mathrm{kN})$ & Extreme strength $\sigma_{b}(\mathrm{MPa})$ \\
\hline No. 1 & 7.68 & 46.3248 & 9.82 & 211.98 \\
No. 2 & 7.88 & 48.7689 & 12.34 & 253.06 \\
No. 3 & 8.04 & 50.7695 & 10.35 & 203.93 \\
No. 4 & 8.2 & 52.8103 & 12.79 & 242.19 \\
No. 5 & 8.14 & 52.0403 & 10.86 & 208.61 \\
No. 6 & 8.5 & 56.7452 & 13.41 & 236.27 \\
Mean value & $\mathbf{8 . 0 7}$ & $\mathbf{5 1 . 2 4 3 2}$ & $\mathbf{1 1 . 5 9}$ & $\mathbf{2 2 6 . 0 1}$ \\
\hline
\end{tabular}

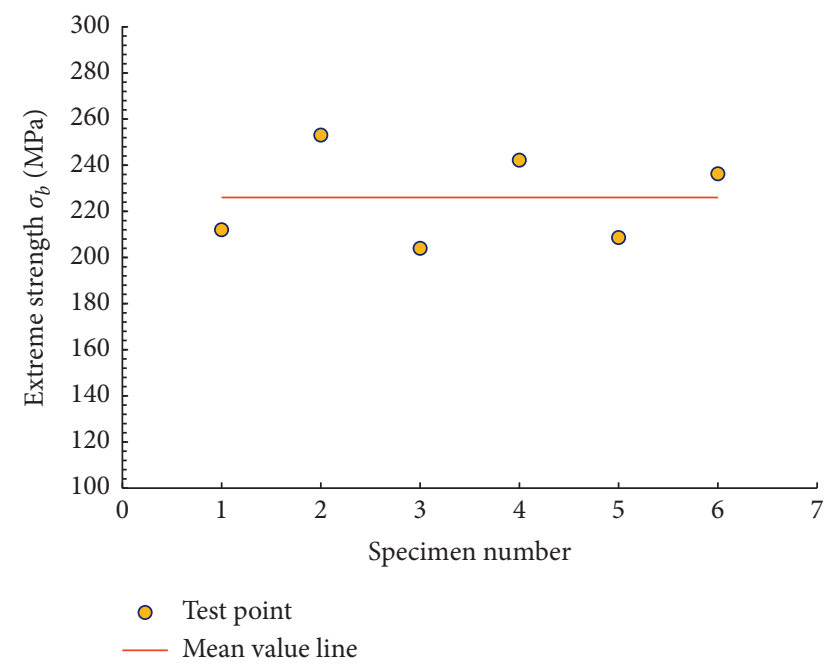

FIgURE 4: Distribution of test values of extreme strength of gray cast iron specimens.

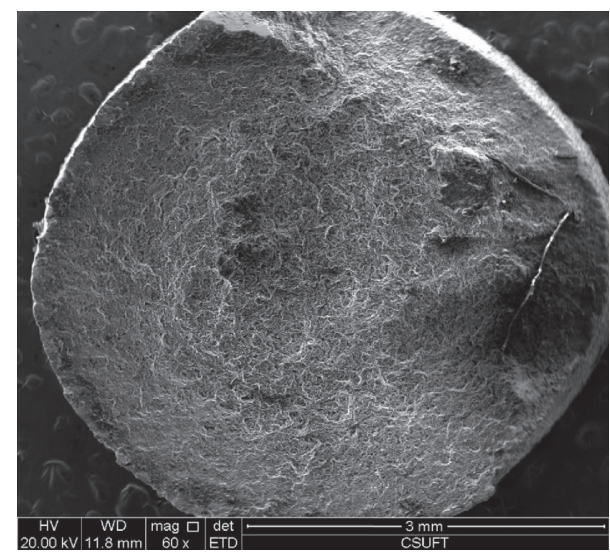

FIgURE 5: Photo of fracture section of gray cast iron specimen.

where $\sigma$ is the applied stress and $\sigma_{0}$ is the mean value of distributed restraining stresses in EDZ. $\mu_{\text {micro }}$ and $\mu_{\text {macro }}$ are the shear modulus, respectively, at the microscale and the macroscale. In fact, $\mu_{\text {micro }}$ should be the shear modulus at the current analytical scale and vary with the shift of the analytical scale denoted by the EDZ length $a . d_{0}$ is the mean size of microstructural grains and $d$ is the length of the crack tip damage zone.

For the static loading failure problem, the loading history can be divided into many small loading steps. The increment of strain energy density factor $S_{\text {micro }}^{\text {macro }}$ in the loading Step $i$ is written as

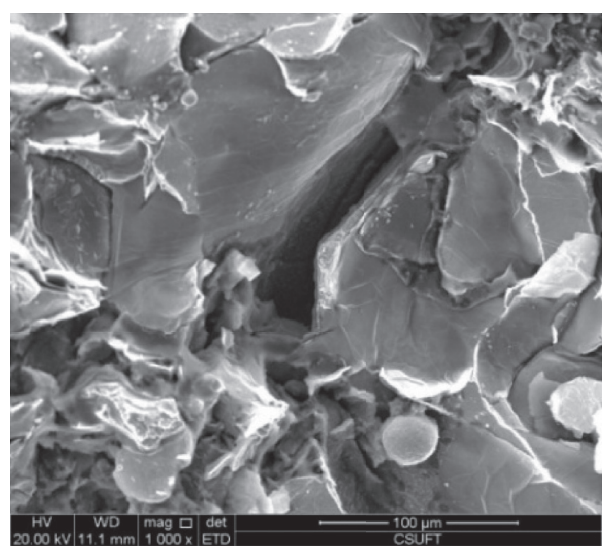

FIGURE 6: Scanned photo of microstructures of gray cast iron HT200 by SEM.

$$
\Delta S_{\text {micro }}^{\text {macro }}=\left(S_{\text {micro }}^{\text {macro }}\right)_{i}-\left(S_{\text {micro }}^{\text {macro }}\right)_{i-1} .
$$

Inserting (1) into (3), it can be obtained that

$$
\Delta S_{\text {micro }}^{\text {macro }}=B a(\Delta \sigma)^{2}(2 i-1) \mu^{*}\left(1-\sigma^{*}\right)^{2} \sqrt{d^{*}} .
$$

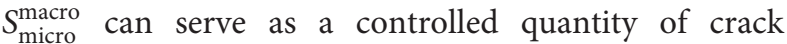
propagation from microscale to macroscale. Therefore, the crack growth rate can be expressed as

$$
\frac{\Delta a}{\Delta N}=C_{0}\left(\Delta S_{\text {micro }}^{\text {macro }}\right)^{m}
$$

where $N$ is the loading step number, and $\Delta N=N_{i}-N_{i-1}$. The substitution (4) into (5) yields

$$
\frac{\Delta a}{\Delta N}=C\left[a(\Delta \sigma)^{2}(2 i-1) \mu^{*}\left(1-\sigma^{*}\right)^{2} \sqrt{d^{*}}\right]^{m},
$$

where $C$ and $m$ are two material parameters, $C=C_{0} B^{m}$.

Three dimensionless parameters $\mu^{*}, \sigma^{*}$, and $d^{*}$ are the functions of generalized time $t$. Here, time $t$ is denoted by the loading step number $N$ or the length $a$ of EDZ. Three functions $\mu^{*}(a), \sigma^{*}(a)$, and $d^{*}(a)$ are the scale transitional functions in the EDZ model that represent the nonequilibrium and nonhomogeneity effects in a failure process. The characteristics of three scale transitional functions $\mu^{*}(a), \sigma^{*}(a)$, and $d^{*}(a)$ have been discussed in detail [11] and are unnecessary to repeat again here.

Numerical integration of (6) can give the $a \sim N$ curve that can be expressed as 


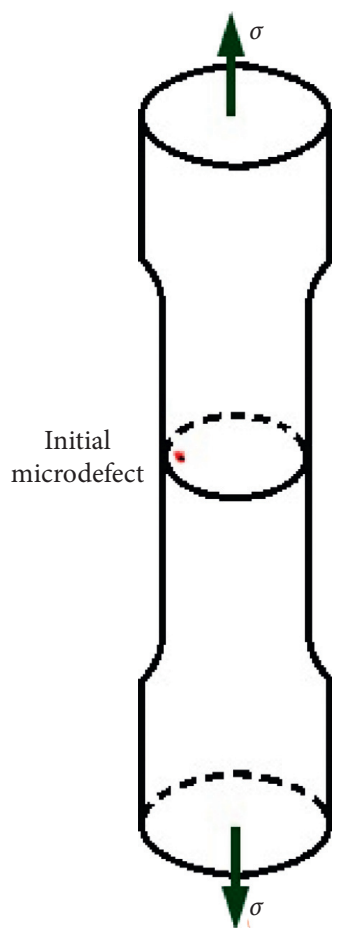

(a)

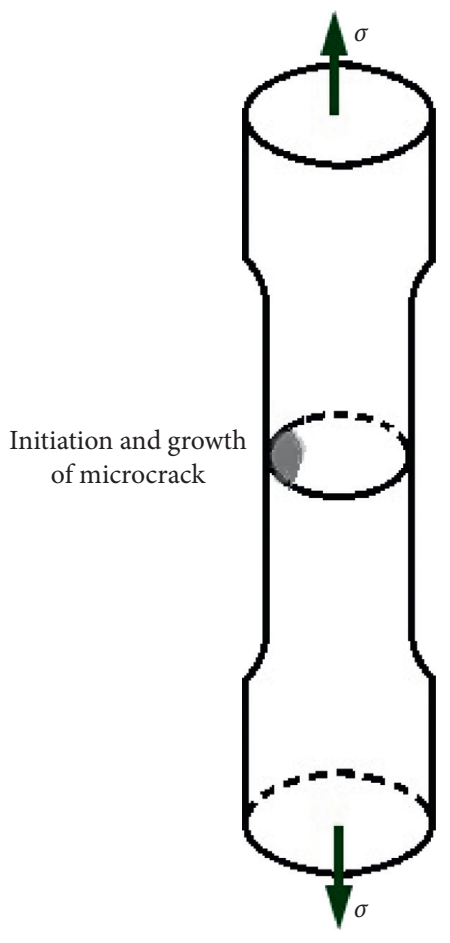

(b)

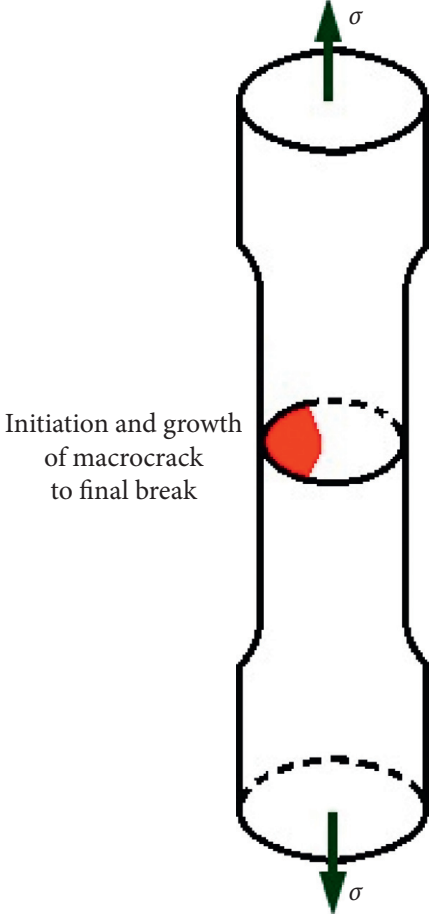

(c)

FIGURE 7: Schematic of fracture process of gray cast iron specimens: (a) initial condition; (b) initiation and growth of microcrack; (c) initiation and growth of macrocrack to the final break.

$\sigma(t)$

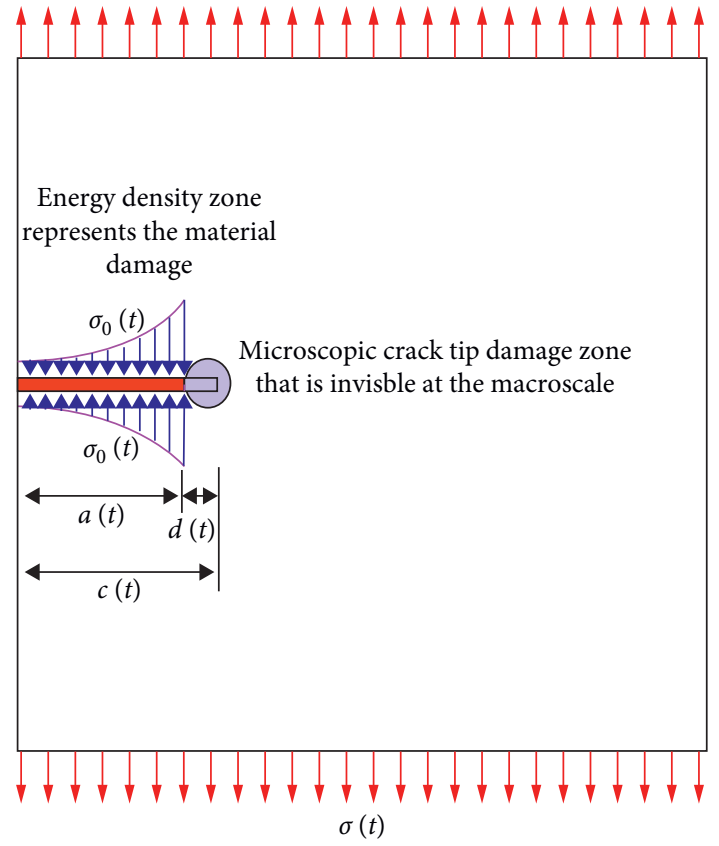

FIGURE 8: Energy density zone model to depict the scale transition of fracture process under the static loading.

$$
\Delta a_{i}=C\left[a_{i-1}(\Delta \sigma)_{i}^{2}(2 i-1) \mu_{i-1}^{*}\left(1-\sigma_{i-1}^{*}\right)^{2} \sqrt{d_{i-1}^{*}}\right]^{m} \Delta N_{i},
$$

$$
\begin{aligned}
\Delta \sigma_{i} & =\sigma_{i}-\sigma_{i-1}, \\
N_{i} & =N_{i-1}+\Delta N_{i},
\end{aligned}
$$$$
\mu_{i-1}^{*}=\mu^{*}\left(a_{i-1}\right) \text {, }
$$$$
\sigma_{i-1}^{*}=\sigma^{*}\left(a_{i-1}\right) \text {, }
$$$$
d_{i-1}^{*}=d^{*}\left(a_{i-1}\right) \text {, }
$$

$$
a_{i}=a_{i-1}+\Delta a_{i}, \quad i=1,2,3, \ldots
$$

This accomplishes the formulation for the failure problem under the static loading condition and it is ready to perform the numerical simulation.

\section{Numerical Simulation and Discussion}

The uniaxial tensile tests of 6 gray cast iron HT200 specimens were carried out, and the results have been given in Table 1 and Figure 4. As discussed earlier, the 
scatter of test data of extreme strength value definitely indicates that the microscopic effects indeed influence the failure behavior and the material strength to some extent even though under the static loading condition. This phenomenon can be precisely simulated by the EDZ model.

For the numerical simulation, three scale transitional functions $\mu^{*}(a), \sigma^{*}(a)$, and $d^{*}(a)$ should be first determined. Referring to Figure 7, the size $a$ is the current analysis scale. The length $a$ gradually increases with the loads while the analysis scale increases accordingly. The length $a$ comes to a macroscopic size while the restraining stresses decrease to zero, a macrocrack appears, and the analysis scale transforms to the macroscale. The initial state is $a=a_{0}$, and $a_{0}$ is the initial microflaw where the fracture process makes onsets. At the initial time, the analysis scale is the scale of microstructures. Therefore, we can assume that $\sigma^{*}=1$ (i.e., no damage), $d^{*}=1$, and $\mu^{*}=3$ at the initial time. When $a>1$, the analysis scale becomes the macroscale. It can be assumed that $\sigma^{*}=0$ (i.e., a macrocrack appears), $d^{*}=8$, and $\mu^{*}=1$. However, the evolution paths of three scale transitional functions cannot be known. Three possible cases A, B, and C are suggested. A parabola function is adopted for Case A with a fast change in the start and a slow change in the end. A parabola function is also assumed for Case $\mathrm{C}$ but with a slow change in the start and a fast change in the end. And, a linear function is used for Case B. Cases A, B, and C are expressed by (11)-(14) and illustrated by Figures 9-11.

Case A (parabola): fast change in the start and slow change in the end:

$$
\left\{\begin{array}{l}
\mu^{*}(a)=1.2 a^{2}-3.2 a+3, \\
\sigma^{*}(a)=0.56 a^{2}-1.56 a+1, \\
d^{*}(a)=-5.6 a^{2}+12.6 a+1, \\
\text { for } a \leq 1 \mathrm{~mm} .
\end{array}\right.
$$

Case B (straight line): a linear change:

$$
\left\{\begin{array}{l}
\mu^{*}(a)=-2 a+3, \\
\sigma^{*}(a)=-a+1, \\
d^{*}(a)=7 a+1, \\
\text { for } a \leq 1 \mathrm{~mm} .
\end{array}\right.
$$

Case C (parabola): slow change in the start and fast change in the end:

$$
\left\{\begin{array}{l}
\mu^{*}(a)=-1.2 a^{2}-0.8 a+3, \\
\sigma^{*}(a)=-0.56 a^{2}-0.44 a+1, \\
d^{*}(a)=5.6 a^{2}+1.4 a+1, \\
\text { for } a \leq 1 \mathrm{~mm} .
\end{array}\right.
$$

When $a>1 \mathrm{~mm}$ indicating that the macrocrack forms and the analysis scale should shift to the macroscale, the values of three functions should be fixed at

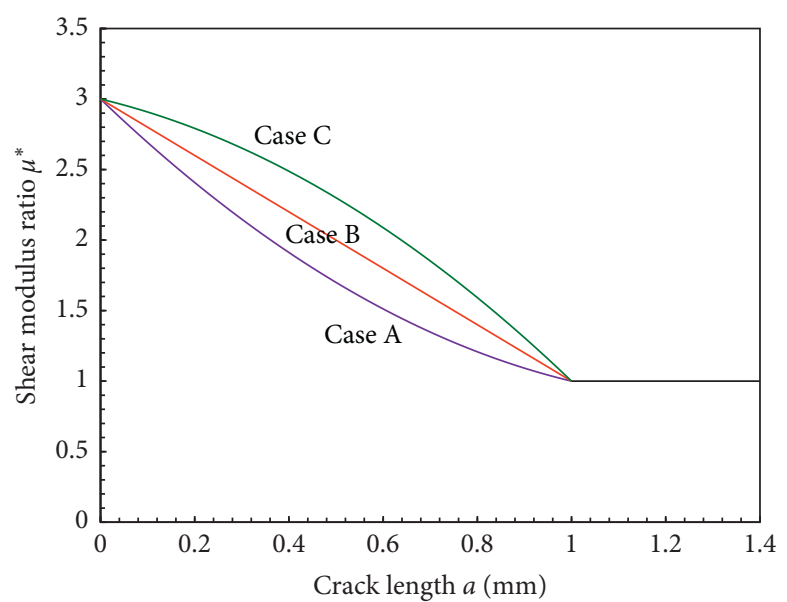

FIGURE 9: Variation of shear modulus ratio $\mu^{*}$ with crack length a.

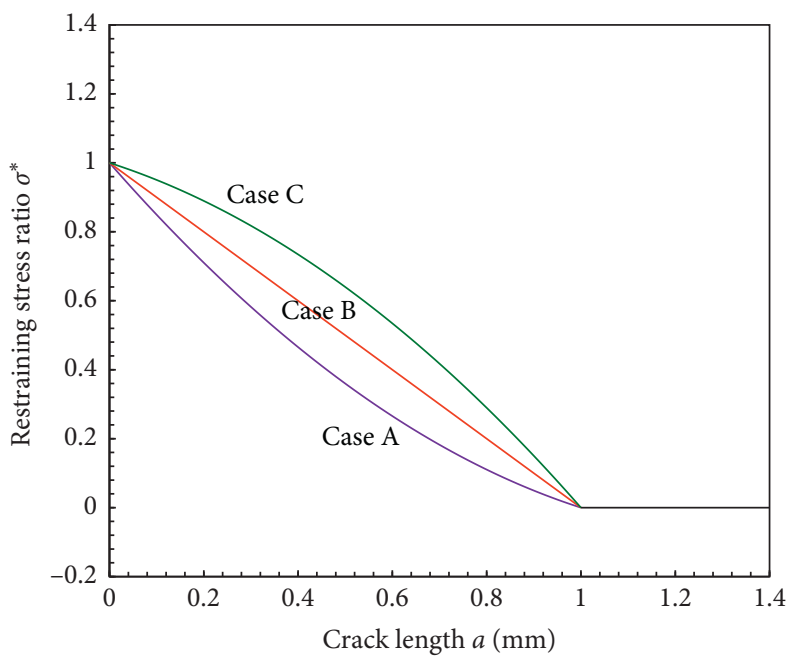

FIgURE 10: Variation of restraining stress ratio $\sigma^{*}$ with crack length a.

$$
\begin{aligned}
\mu^{*} & =1, \\
\sigma^{*} & =0, \\
d^{*} & =8, \\
\text { for } a & \leq 1 \mathrm{~mm} .
\end{aligned}
$$

The test mean value of extreme strength of six specimens is used to determine two other material parameters $C$ and $m$ in (6). In view of Figure 6 , the initial size $a_{0}$ is, $a_{0}=0.1 \mathrm{~mm}$. Take the calculation step $\Delta N=1$ (same step length is adopted), and $\Delta \sigma=0.1 \mathrm{MPa}$. The test mean value of extreme strength is $226.01 \mathrm{MPa}$ (see Table 1) corresponding to the loading step number $N=2260$. After a targeting process, the values of $C$ and $m$ are determined and listed in Table 2 .

Now, all unknown quantities in the model have been determined. The values of extreme strength of six gray cast iron HT200 specimens are calculated and the results are listed in Table 3. It is seen that the calculated values of extreme strength are identical to the test values. The test data can be accurately reproduced by the EDZ model. Moreover, 


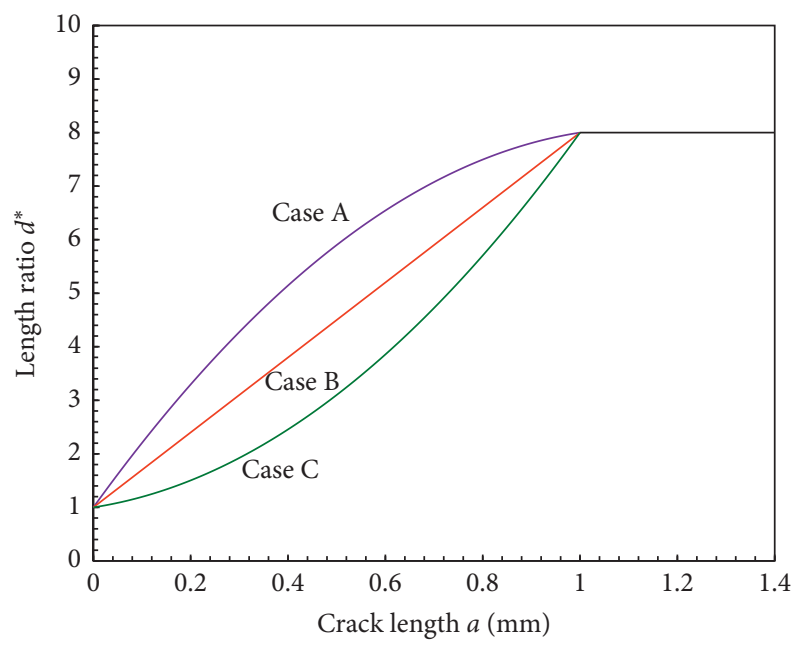

FIgURE 11: Variation of length ratio $d^{*}$ with crack length $a$.

TABLE 2: Values of two parameters $C$ and $m$ in the EDZ model.

\begin{tabular}{lccc}
\hline Model parameters & Case A & Case B & Case C \\
\hline$C$ & $6.490 \times 10^{-5}$ & $8.919 \times 10^{-5}$ & $2.014 \times 10^{-4}$ \\
$m$ & 0.9247 & 0.9247 & 0.9247 \\
\hline
\end{tabular}

TABLE 3: Calculated values of extreme strength of gray cast iron HT200 specimens.

\begin{tabular}{lcccc}
\hline Specimen number & Diameter $\Phi(\mathrm{mm})$ & Initial microflaw size $a_{0}(\mathrm{~mm})$ & Test value $\sigma_{b}(\mathrm{MPa})$ & Calculated value $\sigma_{b}(\mathrm{MPa})$ \\
\hline No. $\mathbf{1}$ & 7.68 & 0.1236 & 211.98 & 211.90 \\
No. 2 & 7.88 & 0.0711 & 253.06 & 253.00 \\
No. 3 & 8.04 & 0.1422 & 203.93 & 203.90 \\
No. 4 & 8.20 & 0.0808 & 242.19 & 242.20 \\
No. $\mathbf{5}$ & 8.14 & 0.1309 & 208.61 & 208.60 \\
No. $\mathbf{6}$ & 8.50 & 0.0870 & 236.27 & 236.30 \\
\hline
\end{tabular}

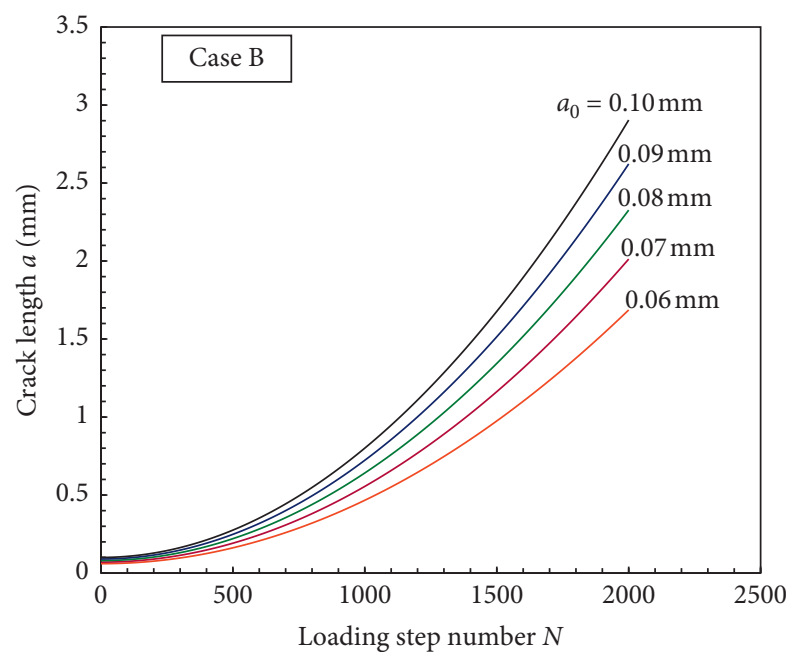

FIGURE 12: Crack length $a$ versus loading step number $N$ curves for different initial microflaw sizes $a_{0}$.

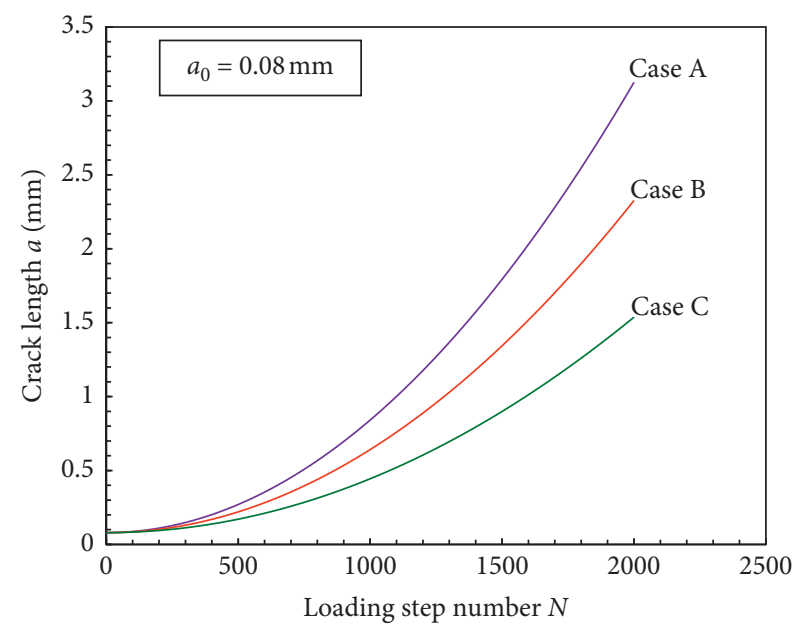

FIGURE 13: Crack length $a$ versus loading step number $N$ curves for different evolution paths of three scale transitional functions $\mu^{*}(a)$, $\sigma^{*}(a)$, and $d^{*}(a)$. 
the scatter of test data can also be precisely simulated by the EDZ model in which the microscopic effects are taken into account.

\section{Microscopic Effect Investigation}

Microscopic effects are represented by the initial microflaw size $a_{0}$ and three scale transitional functions $\mu^{*}(a), \sigma^{*}(a)$, and $d^{*}(a)$ in the EDZ model. Case B is used, and different $a \sim N$ curves under different values of $a_{0}$ are plotted in Figure 12. Obviously, the initial microflaw size $a_{0}$ has a significant influence on the failure process. Moreover, the initial microflaw size $a_{0}$ is fixed at $a_{0}=0.08 \mathrm{~mm}$. A group of $a \sim N$ curves for different evolutionary histories of three functions $\mu^{*}(a), \sigma^{*}(a)$, and $d^{*}(a)$ are displayed in Figure 13. As expected, the evolution path of three scale functions also strongly influences the failure behaviors.

\section{Conclusion}

(1) Energy density zone (EDZ) model is developed for the static strength problem of gray cast iron material in the framework of strain energy density theory. EDZ model can depict the intrinsic behavior of scale transition from microscale to macroscale in a failure process. Furthermore, the nonequilibrium and nonhomogeneity (NENH) effects can also be simulated in the EDZ model. This is realized by the introduction of three basic scale transitional functions, i.e., $\sigma^{*}(a), \mu^{*}(a)$, and $d^{*}(a)$ that represent three key aspects for material failure problems. Function $\sigma^{*}(a)$ denotes the loading/retraining effects related to the material damage degree. Function $\mu^{*}(a)$ stands for the micro/macromaterial property varying with the analysis scale shift. Function $d^{*}(a)$ is related to the micro/macrogeometries

(2) The EDZ model is used to accurately reproduce the ultimate tensile strength test value of gray cast iron HT200 under static load, and compared with the test results, it is concluded that microscopic factors are the main reason for the divergence of test data

(3) The numerical simulation results show that the initial microdefects and microstructure evolution characteristics have a significant influence on the material failure process

\section{Data Availability}

The datasets used in the present study are available from the corresponding author upon reasonable request.

\section{Conflicts of Interest}

The authors declare that they have no conflicts of interest.

\section{References}

[1] Y. C. Lin, J. Deng, Y. Q. Jiang et al., "Hot tensile deformation behaviors and fracture characteristics of a typical Ni-based superalloy," Materials \& design, vol. 55, pp. 949-957, 2014.
[2] Y. C. Lin, J. Deng, Y.-Q. Jiang, D.-X. Wen, and G. Liu, "Effects of initial $\delta$ phase on hot tensile deformation behaviors and fracture characteristics of a typical Ni-based superalloy," Materials Science and Engineering: A, vol. 598, pp. 251-262, 2014.

[3] Y. C. Lin, X. H. Zhu, W. Y. Dong et al., "Effects of deformation parameters and stress triaxiality on the fracture behaviors and microstructural evolution of an Al-Zn-Mg-Cu alloy - science direct," Journal of Alloys and Compounds, vol. 832, Article ID 154988, 2020.

[4] K. Aslantas, S. Talas, and S. Tasgetiren, "Fracture of a compressor rotor made from grey cast iron," Engineering Failure Analysis, vol. 11, no. 3, pp. 369-373, 2004.

[5] G. Bertolino, E. Juan, and Perez-Ipiña, "Geometrical effects on lamellar grey cast iron fracture toughness," Journal of $\mathrm{Ma}$ terials Processing Technology, vol. 179, no. 1-3, pp. 202-206, 2006.

[6] K. L. Fan, G. Q. He, M. She et al., "High-temperature low cycle fatigue behavior of a gray cast iron," Materials Characterization, vol. 98, pp. 37-46, 2014.

[7] M. Metzger and T. Seifert, "Computational assessment of the microstructure-dependent plasticity of lamellar gray cast iron - part II: initial yield surfaces and directions," International Journal of Solids and Structures, vol. 66, pp. 194-206, 2015.

[8] O. Oloyede, T. D. Bigg, R. F. Cochrane, A. M. Mullis, and Mullis, "Microstructure evolution and mechanical properties of drop-tube processed, rapidly solidified grey cast iron," Materials Science and Engineering: A, vol. 654, pp. 143-150, 2016.

[9] F. Berto, D. A. Cendón, and M. Elices, "Fracture behavior under torsion of notched round bars made of gray cast iron," Theoretical and Applied Fracture Mechanics, vol. 84, pp. 157-165, 2016.

[10] L. Collini, G. Nicoletto, and R. Konečná, "Microstructure and mechanical properties of pearlitic gray cast iron," Materials Science and Engineering: A, vol. 488, no. 1-2, pp. 529-539, 2008.

[11] P. Baicchi, L. Collini, and E. Riva, "A methodology for the fatigue design of notched castings in gray cast iron," Engineering Fracture Mechanics, vol. 74, no. 4, pp. 539-548, 2007.

[12] W. Xu, M. Ferry, and Y. Wang, "Influence of alloying elements on as-cast microstructure and strength of gray iron," Materials Science and Engineering: A, vol. 390, no. 1-2, pp. 326-333, 2005.

[13] G. C. Sih, "Implication of scaling hierarchy associated with nonequilibrium: field and particulate," Theoretical and Applied Fracture Mechanics, vol. 37, no. 1-3, pp. 335-369, 2001.

[14] G. C. Sih, "Scalability and homogenization of transitional functions: effects of non-equilibrium and non-homogeneity," Theoretical and Applied Fracture Mechanics, vol. 71, pp. 1420, 2014.

[15] G. C. Sih and X. S. Tang, "Dual scaling damage model associated with weak singularity for macroscopic crack possessing a micro/mesoscopic notch tip," Theoretical and Applied Fracture Mechanics, vol. 42, no. 1, pp. 1-24, 2004.

[16] G. C. Sih and X. S. Tang, "Simultaneity of multiscaling for macro-meso-micro damage model represented by strong singularities," Theoretical and Applied Fracture Mechanics, vol. 42, no. 3, pp. 199-225, 2004.

[17] X. S. Tang and G. C. Sih, "Weak and strong singularities reflecting multiscale damage: micro-boundary conditions for free-free, fixed-fixed and free-fixed constraints," Theoretical and Applied Fracture Mechanics, vol. 43, no. 1, pp. 5-62, 2005. 
[18] G. C. Sih and X. S. Tang, "Screw dislocations generated from crack tip of self-consistent and self-equilibrated systems of residual stresses: atomic, meso and micro," Theoretical and Applied Fracture Mechanics, vol. 43, no. 3, pp. 261-307, 2005.

[19] G. C. Sih and X. S. Tang, "Scaling of volume energy density function reflecting damage by singularities at macro-, mesoand microscopic level," Theoretical and Applied Fracture Mechanics, vol. 43, no. 2, pp. 211-231, 2005.

[20] G. C. Sih and X. S. Tang, "Triple scale segmentation of nonequilibrium system simulated by macro-micro-atomic line model with mesoscopic transitions," Theoretical and Applied Fracture Mechanics, vol. 44, no. 2, pp. 116-145, 2005.

[21] X. S. Tang and G. C. Sih, "Edge dislocations generated from a microcrack under initial residual stress of non-uniform distribution," Theoretical and Applied Fracture Mechanics, vol. 44, no. 3, pp. 208-233, 2005.

[22] G. C. Sih and X. S. Tang, "Simultaneous occurrence of double micro/macro stress singularities for multiscale crack model," Theoretical and Applied Fracture Mechanics, vol. 46, no. 2, pp. 87-104, 2006.

[23] G. C. Sih and X. S. Tang, "Asymptotic micro-stress field dependency on mixed boundary conditions dictated by micro-structural asymmetry: mode I macro-stress loading," Theoretical and Applied Fracture Mechanics, vol. 46, no. 1, pp. 1-14, 2006.

[24] X. S. Tang and C. H. Gao, "Macro-micro dual scale crack model linked by a restraining stress zone with a linear distribution," Theoretical and Applied Fracture Mechanics, vol. 71, no. June, pp. 31-43, 2014.

[25] X. Tang, "Scatter of fatigue data owing to material microscopic effects," Science China Physics, Mechanics and Astronomy, vol. 57, no. 1, pp. 90-97, 2014.

[26] X. S. Tang and T. T. Wei, "Microscopic inhomogeneity coupled with macroscopic homogeneity: a localized zone of energy density for fatigue crack growth," International Journal of Fatigue, vol. 70, pp. 270-277, 2015.

[27] X. S. Tang and X. L. Peng, "An energy density zone model for fatigue life prediction accounting for non-equilibrium and non-homogeneity effects," Theoretical and Applied Fracture Mechanics, vol. 79, pp. 105-112, 2015.

[28] J. R. Zhang, L. Fan, and X. S. Tang, "Energy density zone model and fatigue life prediction considering microscopic effects," Fatigue \& Fracture of Engineering Materials \& Structures, vol. 39, no. 12, pp. 1542-1556, 2016. 